A. R. Heller ${ }^{1}$
M. P. Müller ${ }^{1}$
M. D. Frank ${ }^{1}$
J. Dreßler ${ }^{2}$

\section{Rigor mortis - ein sicheres Todeszeichen?}

Rigor mortis - a Definite Sign of Death?
Zusammenfassung

In der jüngsten Vergangenheit wird in der Bundesrepublik eine kontroverse Diskussion über die Erfüllbarkeit rechtsmedizinischer Qualitätsansprüche an die Leichenschau in der ärztlichen Praxis geführt.

Wir berichten den Fall einer 90-jährigen Frau, die bei suizidaler Benzodiazepin-Überdosierung nach unbekannter Liegezeit in Halblinksseitenlage aufgefunden wurde. Bei der Inspektion der Patientin zeigten sich wegdrückbare fleckförmige Hauterscheinungen an Unterschenkel und Unterarm links. Gleichzeitig fiel ein Rigor (mortis?) des linken Armes auf, der eine Streckung im Ellenbogengelenk verhinderte.

Die stark unterkühlte ateminsuffiziente Patientin wurde intubiert. Eine mechanische kardiopulmonale Reanimation erfolgte bei sehr schwach tastbarem zentralem Puls (RR $70 \mathrm{~mm} \mathrm{Hg}$ systol.) und einer Sinusbradykardie (45/min) mit einzelnen VES nicht. Nach Anbringen einer Venenverweilkanüle (V. jugularis ext. rechts $17 \mathrm{G}$ ) konnte die hämodynamische Situation durch fraktionierte Adrenalin-Gabe und Pufferung mit Bicarbonat stabilisiert werden. In dem Maße, in dem stabile Kreislaufverhältnisse etabliert werden konnten, verschwanden sowohl die Hauterscheinungen als auch die beobachtete Starre der Extremitäten. Im vorliegenden Fall wurde trotz vorhandener Livores und einem auf die linke Körperhälfte begrenzten Rigor ein zentraler Minimalkreislauf vorgefunden, der durch entsprechende Therapie stabilisiert werden konnte. Pathophysiologisch muss eine zentripetal voranschreitende Glykogen- und ATP-Verarmung (Rigor) der Patientin im Rahmen der peripheren Minderperfusion (Livores) in Kombination mit lokalen Kälteerscheinungen (Kälteery-
Abstract

In the past years an ongoing controversial debate exists in Germany, regarding quality of the coroner's inquest and declaration of death by physicians.

We report the case of a 90-year old female, who was found after an unknown time following a suicide attempt with benzodiazepine. The examination of the patient showed livores (mortis?) on the left forearm and left lower leg. Moreover, rigor (mortis?) of the left arm was apparent which prevented arm flexion and extension.

The hypothermic patient with insufficient respiration was intubated and mechanically ventilated. Chest compressions were not performed, because central pulses were (hardly) palpable and a sinus bradycardia $45 / \mathrm{min}$ (AV-block $2^{\circ}$ and sole premature ventricular complexes) was present. After placement of an intravenous line ( $17 \mathrm{G}$, external jugular vein) the hemodynamic situation was stabilized with intermittent boli of epinephrine and with sodium bicarbonate. With improved circulation livores and rigor disappeared.

In the present case a minimal central circulation was noted, which could be stabilized, despite the presence of certain signs of death (livores and rigor mortis). Considering the finding of an abrogated peripheral perfusion (livores), we postulate a centripetal collapse of glycogen and ATP supply in the patients left arm (rigor), which was restored after resuscitation and reperfusion. Thus, it appears that livores and rigor are not sensitive enough to exclude a vita minima, in particular in hypothermic patients with intoxications. Consequently a careful $\mathrm{ABC}$-check should be performed even in the presence of apparently certain 
them, Kältestarre) postuliert werden, die nach Reperfusion wieder aufgehoben wurden.

Damit erscheint die Sensitivität und Spezifität von fleckförmigen Hauterscheinungen und Muskelstarre für die Todesfeststellung insbesondere bei Intoxikationen und unterkühlten Patienten nicht ausreichend. Ein sorgfältiger $A B C-C h e c k$ sollte auch bei scheinbarem Vorliegen sicherer Todeszeichen durchgeführt werden, um das Vorhandensein einer Vita reducta nicht zu übersehen. Apparative Zusatzdiagnostik wie EKG könnte die Rate falsch positiver Todesfeststellungen reduzieren.

Inwiefern der Beginn von kardiopulmonalen Reanimationsmaßnahmen durch medizinisches Assistenzpersonal (Rettungsassistenten etc.) auch bei Vorliegen von Rigor und Livores bis zum Eintreffen des Notarztes zu fordern ist, muss kritisch diskutiert werden.

\section{Schluisselwörter}

Totenstarre $\cdot$ Leichenflecken $\cdot$ Vita minima $\cdot$ Benzodiazepin $\cdot$ Intoxikation

\section{Einleitung}

In jüngster Vergangenheit wird in bundesdeutschen berufspolitischen Fachblättern eine kontroverse Diskussion über die Erfüllbarkeit rechtsmedizinischer Qualitätsansprüche an die Leichenschau in der ärztlichen Praxis geführt $[3,7,8]$ und die Frage aufgeworfen, ob die Bestellung eines amtlichen Leichenbeschauers nach britischem Vorbild dieses Dilemma zu lösen im Stande sei [1]. Neben der Feststellung der Todesursache, der Todesart, der Todeszeit sowie übertragbarer Krankheiten und dem Nachkommen entsprechender Meldepflichten obliegt dem Arzt jedoch zuallererst die sichere Feststellung des eingetretenen Todes. Hierbei haben die sicheren Todeszeichen (Tab.1) besondere Wertigkeit [6]. Eine Kombination von Medikamentenintoxikation mit allgemeiner Unterkühlung ist jedoch die häufigste Ursache für eine Vita minima und Vita reducta mit fälschlicher Todesannahme [6].

\section{Fallbericht}

An einem Apriltag (Außentemperatur ca. $12{ }^{\circ} \mathrm{C}$ ) erfolgte um 9.27 Uhr der Notruf unter dem Einsatzstichwort „Tablettenintoxikation“ über eine Hausarztpraxis an die Rettungsleitstelle. Wegen mangelnder Verfügbarkeit eines Notarztes im Kreisgebiet erfolgte die Alarmierung eines RTW, die primär von Nachbarn alarmierte Hausärztin fuhr ebenfalls zum Notfallort. Der zu diesem Zeitpunkt im Kreisgebiet im Einsatz befindliche RTH Christoph 38 erhielt ebenfalls diesen Auftrag und traf um 9.46 Uhr bei der Patientin ein.

Im Schlafzimmer der alleinstehenden unter Depressionen leidenden 90-Jährigen, die ansonsten keine relevanten Vorerkrankungen bot, zeigte sich das typische Bild einer Schlafmittel- Intoxikation. Die ca. $50 \mathrm{~kg}$ schwere Patientin lag mit grauem Hautkolorit in Halbseitenlage links vor ihrem Bett, in der Nähe je eine geöffnete Packung Lorazepam und Zopiclon, aus denen jeweils 10 Tabletten fehlten. An Arm und Unterschenkel links fielen rote signs of death, to avoid underdiagnosing a vita minima. Additional ECG-monitoring is required to reduce the rate of false positive declarations of death. To what extent basic life support by paramedics should commence when rigor and livores are present until physician DNR order, deserves further discussion.

\section{Key words}

Rigor mortis · livores $\cdot$ vita minima $\cdot$ benzodiazepine $\cdot$ intoxication

fleckförmige nicht konfluierende Verfärbungen der Haut auf, und der linke Arm zeigte eine Starre, die die Streckung des gebeugten Ellenbogengelenks verhinderte.

\section{Befund}

Bei der körperlichen Untersuchung der bewusstlosen, minimal atmenden, stark unterkühlten Patientin zeigte sich ein sehr schwacher zentraler Puls. Im EKG ließ sich eine Sinusbradykardie (45/min) mit AV-Block $2^{\circ}$ (Wenckebach) und einzelne ventrikuläre Extrasystolen ableiten. Bei insuffizienter Zirkulation und Spontanatmung war eine pulsoxymetrische Sauerstoffsättigung peripher nicht messbar. Die Pupillen waren beidseits weit und lichtstarr. Im Rahmen der primären Wiederbelebungsmaßnahmen erfolgte die Intubation. Auf die mechanische kardiopulmonale Reanimation wurde bei spontanen systolischen Blutdrücken um $70 \mathrm{~mm} \mathrm{Hg}$ verzichtet.

\section{Therapie}

Zur hämodynamisch stabilisierenden Katecholamintherapie wurde eine Venenverweilkanüle $(17 \mathrm{G})$ in die V. jugularis ext. rechts eingelegt. Periphere Venenpunktionen waren wegen der Starre des linken Armes, die eine Streckung im Ellenbogengelenk verhinderte, und ungünstiger Venenverhältnisse des rechten $\mathrm{Ar}$ mes nicht erfolgreich. Zudem konnte bei der unklaren peripheren Perfusionssituation nicht von einer sicheren systemischen Wirkung der peripher applizierten Medikamente ausgegangen werden. Die Zirkulation konnte mit 5 minütigen Boli von $0,2 \mathrm{mg}$ Adrenalin (gesamt $3 \mathrm{mg}$ ), $2 \times 0,5 \mathrm{mg}$ Atropin sowie blinder Azidosekorrektur mit Natriumbicarbonat (100 mval) und Volumentherapie (1000 ml Ringerlösung) medikamentös stabilisiert werden. In dem Maße, in dem stabile Kreislaufverhältnisse etabliert werden konnten, kehrte eine normale Hautfarbe zurück, und es verschwanden sowohl die fleckförmigen Hautverfärbungen als auch die beobachtete Starre der abhängigen Extremitäten (links). 
Im weiteren Verlauf trat intermittierend ein Herdblick nach rechts oben sowie spontane Kaubewegungen auf. Es erfolgte eine fraktionierte Analgesie mit Fentanyl 0,3 mg sowie die Relaxierung mit $6 \mathrm{mg}$ Pancuronium. Der Versuch einer Antidottherapie mit 0,5 mg Flumazenil zeigte zu diesem Zeitpunkt erwartungsgemäß keine Auswirkung auf die Bewusstseinslage.

Unter fortgesetzter Katecholamin- und Volumentherapie konnte die Patientin in Reanimationsbereitschaft bodengebunden in das $21 \mathrm{~km}$ entfernte Kreiskrankenhaus gebracht werden (Arztbegleiteter RTW-Transport 10.07 - 10.25 Uhr). Ein Lufttransport im eingesetzten Hubschraubertyp BO 105 CBS, in dem während des Fluges eine effektive kardiopulmonale Reanimation nicht möglich ist, wurde bei der hämodynamisch instabilen Patientin trotz eines annehmbaren Zeitvorteils (Landung am Zielkrankenhaus bereits um 10.19 Uhr) in der konkreten Lage abgelehnt.

Aufgrund der eingetretenen Rhabdomyolyse und Laktazidose im Rahmen der Liegezeit (annehmbar 6-8h) wurde die hämodynamische Situation der Patientin nach initialer Besserung zunehmend instabil, so dass sie innerhalb von $8 \mathrm{~h}$ verstarb.

\section{Diskussion}

Das Vorliegen von unsicheren Todeszeichen (Tab.1) rechtfertigt nicht die Annahme des Todes, sondern ist vielmehr die Indikation zum Beginn entsprechender Reanimationsmaßnahmen [12]. Die Feststellung des eingetretenen biologischen Todes wird bei der Leichenschau jedoch als unproblematisch dargestellt, wenn sichere Todeszeichen (Tab. 1) zu finden sind [6]. Das Vorhandensein solcher Befunde schließt das Vorliegen eines sog. Scheintodes (Vita minima bzw. reducta) aus. Reale Ursachen für einen Scheintod-ähnlichen Zustand können z. B. bestehende Alkoholvergiftungen, Epilepsien, Schädel-Hirn-Traumata, Intoxikationen oder Komata unterschiedlicher Genese sein [6]. Ist für den Leichenschau haltenden Arzt ein sicheres Todeszeichen sichtbar, ist der Individualtod mit Sicherheit eingetreten.

Nach dem Herz-Kreislauf-Stillstand ist unter normalen Umständen schon nach 15-20 min mit dem Auftreten von Leichenflecken zu rechnen, während sich eine Leichenstarre, beginnend am Kiefergelenk üblicherweise erst nach $2-4 \mathrm{~h}$ in kraniokaudaler Richtung meist symmetrisch ausbreitet und nach 6-8 h am gesamten Körper voll ausgeprägt ist. Pathophysiologisches Korrelat ist eine Dehydratation und Bildung eines starren Aktomyosingels der Myofibrillen bei Fehlen von ATP. Typischerweise sind dabei mehrere Gelenke versteift. Demnach reicht die Untersuchung eines einzelnen Gelenkes nicht aus, um einen Rigor mortis zweifelsfrei zu diagnostizieren.

Im vorliegenden Fall ergab sich somit scheinbar eine Diskrepanz zwischen den „sicheren Todeszeichen“ Rigor mortis des linken Armes und wegdrückbaren Livores an den abhängigen Extremitäten links und den lichtstarren Pupillen sowie der vorhandenen Vita minima (schwache Atmung und schwacher Puls), die bei oberflächlicher Untersuchung nicht erkannt worden wäre. Insbesondere die apparative EKG-Diagnostik war hier bei fehlender pulsoxymetrisch gemessener peripherer Sauerstoffsättigung richtungweisend.

Bei genauer Betrachtung der vorgefundenen Befunde erfüllte die isolierte Bewegungseinschränkung im linken Ellenbogengelenk sowie am linken Bein die Kriterien eines Rigor mortis nicht. Zudem kommt es bei kalter Umgebungstemperatur - wie im vorliegenden Fall - in der Regel zur erheblichen Verzögerung des Eintritts der Totenstarre, so dass aus rechtsmedizinischer Sicht Zweifel daran bestehen, ob bei der beschriebenen Starre ante mortem wirklich ein Rigor mortis im Sinne der Definition vorlag. Vielmehr könnte es sich hierbei um eine Kältestarre der abhängigen Extremitäten (Linksseitenlage) gehandelt haben, die in ummittelbarem Kontakt mit dem kalten Fußboden standen und daher am stärksten von der Wärmeabgabe betroffen waren. Eine Kältestarre kann insbesondere bei vorhandener Vita minima in Temperaturbereichen unter $28^{\circ} \mathrm{C}$ auftreten und ist ein charakteristisches Symptom für die Hypothermie im Lähmungsstadium.

Aufgrund der erforderlichen Wiederbelebungsmaßnahmen Intubation, Überwachung, repetetive Katecholamin-Gabe etc. wurde präklinisch bei der offensichtlich unterkühlten Patientin eine niedrige Priorität für die Messung der Körpertemperatur festgesetzt. Die Kenntnis einer exakten rektalen Temperatur hätte das therapeutische Vorgehen zu diesem Zeitpunkt nicht beeinflusst. Schließlich wurde bei der instabilen Patientin auf eine Temperaturmessung zugunsten eines zügigen Transportes ganz verzichtet. Die Intensivstationskurve weist um $11.00 \mathrm{Uhr}$ eine Körpertemperatur von $30,1^{\circ} \mathrm{C}$ aus.

Ähnlich wie bei der Interpretation der aufgehobenen Armbeweglichkeit als Rigor mortis sind ebenso Zweifel bei der Auslegung der fleckförmigen Hautverfärbungen als Livores mortis angebracht. Totenflecke sind im rechtsmedizinischen Sinne Folge des irreversiblen Herz-Kreislauf-Stillstandes. Bei Totenflecken wird unterschieden zwischen der Frühphase mit fleckförmiger Verfär-

Tab. 1 Symptome, die im Rahmen einer Todesfeststellung abzugrenzen sind

\begin{tabular}{|c|c|c|}
\hline $\begin{array}{l}\text { sichere Todeszeichen } \\
\text { biologischer Tod }\end{array}$ & $\begin{array}{l}\text { unsichere Todeszeichen } \\
\text { klinischer Tod }\end{array}$ & $\begin{array}{l}\text { sonstige Symptome } \\
\text { keine Todeszeichen }\end{array}$ \\
\hline $\begin{array}{l}\text { - Totenflecke (Livores mortis) } \\
\text { - Totenstarre (Rigor mortis) } \\
\text { - fortgeschrittene Leichenerscheinungen } \\
\text { (Autolyse, Fäulnis, Verwesung) } \\
\text { - Körperzerstörung, die nicht mit dem Leben } \\
\text { vereinbar ist }\end{array}$ & $\begin{array}{l}\text { - Bewusstlosigkeit } \\
\text { - Atemstillstand } \\
\text { - fehlende Herztätigkeit, kein Puls } \\
\text { - Areflexie } \\
\text { - Lichtstarre, weite Pupillen, } \\
\text { fehlende Pupillenreaktion } \\
\text { - Absinken der Körpertemperatur }\end{array}$ & $\begin{array}{l}\text { - Rigor = gesteigerte Grundspannung der Skelett- } \\
\text { muskulatur (Agonisten und Antagonisten) } \\
\text { Z. B. M. Parkinson } \\
\text { - Cutis marmorata congenita } \\
\text { - lokale Kälteschäden (Kältestarre, Kälteerythem) }\end{array}$ \\
\hline
\end{tabular}


bung der Haut, bedingt dadurch, dass sich die Kapillarbezirke der Haut zunächst nicht gleichmäßig, sondern arealweise mit Blut füllen, und den konfluierenden Totenflecken, die ein Endzustand des hypostatischen Absackens des Blutes in tiefer gelegene Körperregionen darstellen. Bis zum Eintritt der Hämokonzentration durch postmortalen Wasserverlust sind Livores wegdrückbar bzw. auch umlagerbar. Diese Hämokonzentration ist erfahrungsgemäß nach einer Leichenliegezeit von 12 Stunden eingetreten. In der rechtsmedizinischen Literatur wird darauf hingewiesen, dass in seltenen Fällen bei langer Agonie altersschwacher Patienten durch die nachlassende Herzkraft bereits vor dem Tode „totenfleckartige Hautverfärbungen“ auftreten, die im Volksmund als „Kirchhofrosen“ bezeichnet werden. Im vorliegenden Fall muss ein Kälteerythem als Symptom eines zweitgradigen lokalen Kälteschadens differenzialdiagnostisch abgegrenzt werden. Solche Hauterscheinungen imponieren als braunrot-ziegelrote Erythemzonen bevorzugt an Akren, Knie- und Ellenbogenregion (Zonen geringer Vasularisation). Unwahrscheinlicher in diesem Zusammenhang ist eine Cutis marmorata (teleangiectatica congenita).

Im Rahmen der vorliegenden Intoxikation muss insgesamt von einer erheblichen Liegezeit (ca. 6-8 h) mit bestehender Vita minima ausgegangen werden. Dabei kam es bei langsam fortschreitender Zentralisierung des Kreislaufes in Kombination mit lokalen Kältewirkungen bereits zu einem peripheren Perfusionsstopp mit Substratmangel, der zu den totenfleckartigen Hautverfärbungen und der isolierten Starre der abhängigen Extremitäten geführt hat, obwohl immer noch eine zentrale Minimalzirkulation erhalten war.

Als Gründe für die Leichenstarre wird allgemein eine Depletion der Muskulatur von Glykogen und ATP als Energielieferanten angenommen $[4,9]$. Daher ist die Eintrittsgeschwindigkeit und Stärke des Rigor mortis von dem unmittelbar vor Todeseintritt verfügbaren ATP im Muskel abhängig. In diesem Zusammenhang konnte gezeigt werden, dass Ratten, die dekapitiert wurden, einen signifikant langsameren Eintritt der Leichenstarre hatten als solche, die ertrunken sind und unmittelbar vor ihrem Tod noch erhebliche Muskelaktivität und damit ATP-Verbrauch hatten $[5,10]$. Ähnliche Fallberichte existieren, die einen ungewöhnlich schnellen Eintritt der Leichenstarre bei Dauerläufern zeigten, die an einem akuten Myokardinfarkt verstarben. Neben der muskulären Energiereserve scheint die Art der Muskelfasern ebenfalls die Ausprägung des Rigor mortis zu beeinflussen [4], was den üblichen Beginn im Kiefergelenk teilweise erklärt. Im vorliegenden Fall gehörte jedoch die Kiefermuskulatur zu dem zentralen Kompartiment, das noch eine Minimalversorgung (und Temperierung) durch den Kreislauf hatte und somit eine längere ATP und Glykogenreserve aufwies als die Extremitäten, und damit nicht wie gewöhnlich zuerst der Leichenstarre unterlag.

Die nach Restituierung der Zirkulation und Reperfusion bei der Patientin beobachtete Aufhebung der Starre muss so gedeutet werden, dass die erneut verfügbare ATP-Menge hinreichend war, seiner normalen „Weichmacherfunktion“ in der Muskulatur nachzukommen [11]. Entsprechende experimentelle Befunde zeigen eine partielle Rückbildung des Rigor mortis nach ATP-Behandlung [2]. Eine signifikante Erwärmung der abhängigen (linksseitigen) Extremitäten hingegen erscheint im geschilderten Fall eher unwahrscheinlich.

In dieser Kasuistik war eine Intoxikation mit den BenzodiazepinRezeptoragonisten Lorazepam und Zopiclon anzunehmen. Benzodiazepine und die neuen, mit den Benzodiazepinen chemisch nicht verwandten Substanzen Zolpidem und Zopiclon aktivieren selektiv das neuroinhibitorische GABAerge Transmittersystem. Sie haben aber am $G_{A B A}$-Benzodiazepin/Chlorid-Kanal-Rezeptor-Komplex nicht exakt dieselben Bindungsstellen. Trotzdem wirkt Flumazenil als spezifischer Antagonist für alle diese Wirkstoffe. Während Zopiclon einer raschen Resorption und Metabolisierung zu wirksamen Metaboliten unterliegt, ist der Wirkeintritt des hydrophilen Lorazepam eher langsam.

Trotz ihrer Verschreibungspflicht sind Benzodiazepine aufgrund der hohen Verschreibungsfrequenz die häufigste Ursache von akuten Schlafmittelvergiftungen. Atemdepression ist zwar eine bekannte Nebenwirkung dieser Substanzen, jedoch kommt es zu lebensgefährlicher Hypoventilation eher bei Kombinationsvergiftungen mit anderen Sedativa/Hypnotika (z.B. Alkohol, Opioide) oder bei gleichzeitigen kardiopulmonalen Vorerkrankungen. Dabei ist die akute Toxizität von Zopiclon deutlich geringer als diejenige anderer kurz wirksamer Benzodiazepine, wobei Bradykardie und AV-Blockierungen trotzdem beschrieben sind.

Wegen der vermutlich mehrstündigen Liegedauer der Patientin wurde angenommen, dass die überwiegende Menge des Wirkstoffs bereits resorbiert war und evtl. verbliebene Tablettenreste den Magen bereits passiert hatten. Da die kritische kardiopulmonale Situation mit dauernder Katecholaminpflicht im Vordergrund stand, wurde zugunsten eines zügigen Transportes in die nächstgelegene Intensivstation auf eine primäre Giftelimination (Magenspülung) vor Ort verzichtet. Dennoch erfolgte eine spezifische Antiodottherapie mit Flumazenil. Die Patientin war zu diesem Zeitpunkt bereits analgosediert und relaxiert. Daher konnte nicht erwartet werden, dass nach der Antagonisierung Veränderungen der Bewusstseinslage oder Spontanbewegungen auftreten würden. Vielmehr war es das Ziel, eine Benzodiazepin-Nebenwirkung als mögliche Ursache der vorliegenden symptomatischen kardialen Arrhythmien (AV-Block und ventrikuläre Extrasystolen) auszuschalten bzw. einer Aggravierung des kardialen Zustandes vorzubeugen.

Dieser Bericht zeigt, dass die zweifelsfreie Entscheidung über das Vorliegen „sicherer Todeszeichen“ im Einzelfall schwierig sein kann. Das einseitige Vorhandensein eines Rigor und totenfleckartiger Hautverfärbungen sowie beidseits lichtstarrer Pupillen rechtfertigt aber keinesfalls eine Todesannahme und muss zwingend durch andere klinische (sorgfältiger $\mathrm{ABC}-\mathrm{Check}$ ) und apparative Untersuchungsmethoden (EKG) ergänzt werden.

Im Kontext der Diskussion über die Qualität der ärztlichen Leichenschau und der damit im Zusammenhang stehenden möglichen fälschlichen Todesannahmen (Problem der Spezifität) muss gleichzeitig auch mit ärztlichem ethisch-forensischem Augenmaß der Beginn von Maßnahmen der Herz-Lungen-Wiederbelebung bei Patienten mit bereits fortgeschrittenem Sterbeprozess betrachtet werden. Insbesondere muss kritisch diskutiert werden, ob auch von medizinischem Assistenzpersonal in Zwei- 
felsfällen auch beim Vorliegen „sicherer Todeszeichen“ die Durchführung von Basismaßnahmen der Reanimation verlangt werden soll, bis ein Abbruch der Maßnahmen von einem Arzt angeordnet wird.

\section{Literatur}

${ }^{1}$ Christian A. Leichenschau-Wertvoller Bestandteil der hausärztlichen Versorgung. Dt Ärztebl 2003; 100: A3302

${ }^{2}$ Hegarty PV, Heffron JJ. Partial reversal by adenosine triphosphate of the rigor-induced decrease in the diameter of skeletal muscle fibres. Comp Biochem Physiol A 1975; 50: 675-680

${ }^{3}$ Jentsch E. Leichenschau: Fragen. Dt Ärztebl 2001; 98: A2025 - A2026

${ }^{4}$ Kobayashi M, Takatori T, Nakajima M, Saka K, Iwase H, Nagao M, Niiji$\mathrm{ma} \mathrm{H}$, Matsuda Y. Does the sequence of onset of rigor mortis depend on the proportion of muscle fibre types and on intra-muscular glycogen content? Int J Legal Med 1999; 112: 167- 171

${ }^{5}$ Krompecher T, Fryc O. Experimental evaluation of rigor mortis. IV. Change in strength and evolution of rigor mortis in the case of physical exercise preceding death. Forensic Sci Int 1978; 12: $103-107$
${ }^{6}$ Madea B (Hrsg.). Die Ärztliche Leichenschau. Rechtsgrundlagen, praktische Durchführung, Problemlösungen. Berlin, Heidelberg, New York: Springer, 1999: 113-185

${ }^{7}$ Madea B, Dettmeyer R. Ärztliche Leichenschau und Todesbescheinigungen. Dt Ärztebl 2003; 100: A3161 - A3162

8 Merten M. Ärztliche Leichenschau-Qualität Mangelhaft. Dt Ärztebl 2003; 100: A2558

${ }^{9}$ Nauss KM, Davies RE. Changes in phosphate compounds during the development and maintenance of rigor mortis. J Biol Chem 1966; 241: $2918-2922$

${ }^{10}$ Pence HH, Pence S, Kurtul N, Bakan E, Kok AN, Kocoglu H. The alterations in adenosine nucleotides and lactic acid levels in striated muscles following death with cervical dislocation or electric shock. Soud Lek 2003; 48: 8-11

${ }^{11}$ Silbernagl S. Molekulare Mechanismen der Muskelkontraktion. In: Silbernagl S, Despopoulos A (Hrsg.). Taschenatlas Physiologie. Stuttgart: Thieme, 2003: 38-39

12 Wenzel V, Voelckel WG, Krismer AC, Mayr VD, Strohmenger HU, Baubin MA, Wagner-Berger $\mathrm{H}$, Stallinger A, Lindner KH. Die neuen internationalen Richtlinien zur kardiopulmonalen Reanimation. Eine Analyse und Kommentierung der wichtigsten Änderungen. Anaesthesist 2001; 50: $342-357$ 